\title{
Deletion of cg1360 Affects ATP Synthase Function and Enhances Production of L-Valine in Corynebacterium glutamicum ${ }^{\mathrm{S}}$
}

\author{
Xiaochen Wang ${ }^{1}$, Hongyu Yang ${ }^{2}$, Wei $\mathrm{Zhou}^{2,3}$, Jun $\mathrm{Liu}^{2,3}$, and Ning $\mathrm{Xu}^{2,3 *}$ \\ ${ }^{1}$ University of Science and Technology of China, Hefei 230026, P.R. China \\ ${ }^{2}$ Tianjin Institute of Industrial Biotechnology, Chinese Academy of Sciences, Tianjin 300308, P.R. China \\ ${ }^{3}$ Key Laboratory of Systems Microbial Biotechnology, Tianjin Institute of Industrial Biotechnology, Chinese Academy of Sciences, Tianjin \\ 300308, P.R. China
}

Received: April 12, 2019

Revised: July 22, 2019

Accepted: July 25, 2019

First published online

July 30, 2019

*Corresponding author

Phone: +86-22-24828781;

Fax: +86-22-84861926;

E-mail: xu_n@tib.cas.cn

\section{S upplementary data for this} paper are available on-line only at http://jmb.or.kr.

pISSN 1017-7825, eISSN 1738-8872

\section{Copyright(C) 2019 by}

The Korean Society for Microbiology and Biotechnology
Bacterial ATP synthases drive ATP synthesis by a rotary mechanism, and play a vital role in physiology and cell metabolism. Corynebacterium glutamicum is well known as an industrial workhorse for amino acid production, and its ATP synthase operon contains eight structural genes and two adjacent genes, cg1360 and cg1361. So far, the physiological functions of Cg1360 (GenBank CAF19908) and Cg1361 (GenBank CAF19909) remain unclear. Here, we showed that Cg1360 was a hydrophobic protein with four transmembrane helices (TMHs), while no TMH was found in Cg1361. Deletion of $\operatorname{cg} 1360$, but not $\operatorname{cg} 1361$, led to significantly reduced cell growth using glucose and acetic acid as carbon sources, reduced F1 portions in the membrane, reduced ATP-driven proton-pumping activity and ATPase activity, suggesting that Cg1360 plays an important role in ATP synthase function. The intracellular ATP concentration in the $\Delta c g 1360$ mutant was decreased to $72 \%$ of the wild type, while the NADH and NADPH levels in the $\Delta c g 1360$ mutant were increased by $29 \%$ and $26 \%$, respectively. However, the $\Delta \operatorname{cog} 1361$ mutant exhibited comparable intracellular ATP, NADH and NADPH levels with the wild-type strain. Moreover, the effect of cg1360 deletion on L-valine production was examined in the L-valine-producing V-10 strain. The final production of L-valine in the V-10- $\Delta$ cg1360 mutant reached $9.2 \pm 0.3 \mathrm{~g} / 1$ in shake flasks, which was $14 \%$ higher than that of the V-10 strain. Thus, Cg1360 can be used as an effective engineering target by altering energy metabolism for the enhancement of amino acid production in C. glutamicum.

Keywords: ATP synthase, cg1360, C. glutamicum, L-valine production

\section{Introduction}

The ATP synthase is a multi-subunit, membrane-bound enzyme complex that is found in all living organisms. It can rely on the proton electrochemical gradient to drive ADP and Pi to synthesize ATP by a rotational catalytic mechanism, and also hydrolyze ATP to create an ion gradient needed for other physiological activities [1-5]. The intact ATP synthase is typically composed of two major sub-complexes, the membrane-embedded Fo sector and the water-soluble F1 sector. The hydrophobic Fo domain consists of three subunits (a,b, and c) and can function as a proton channel. The hydrophilic F1 domain consists of five subunits $(\alpha, \beta, \gamma, \delta$, and $\varepsilon)$ and has three catalytic sites at the interfaces of $\alpha$ and $\beta$ subunits $[2,6,7]$. In most bacteria, the atp operon contains nine open reading frames (ORFs), including eight structural genes (atpBEFHAGDC) and one preceded atpI gene [8, 9]. AtpI, also known as UncI, is a small membrane protein and is shown to have a role in the c-subunit ring formation during the assembly of some ATP synthases. In Escherichia coli, deletion of the atpI gene only shows a slight reduction in the biomass yield and ATPase activity [10]. Similarly, loss of the atpI gene in Bacillus pseudofirmus OF4 has no apparent effects on the nonfermentative growth [9]. Further investigation shows that AtpI of B. pseudofirmus OF4 is not 
required for the c-ring assembly, but it supports the optimal ATP synthase stability [9]. However, the product of the atpI gene in Propionigenium modestum seems to play a necessary chaperone-like role in the formation of c-rotor ring and the subsequent assembly of ATP synthase [11, 12]. Overall, the physiological significance of AtpI in different species is still ambiguous, and further investigation on other microorganisms will provide more clues into the understanding of bacterial AtpI.

ATP is typically generated from oxidative phosphorylation with the membrane-bound F1Fo-ATP synthase, or produced by substrate level phosphorylation (SLP). The regulation of ATP supply is considered as a promising strategy to improve cellular metabolism for enhanced bioproduction yields [13-15]. The $\mathrm{H}^{+}$-ATPase deficient mutants of E. coli have been successfully applied for increasing the yields of pyruvic acid [16], acetic acid [17], and alanine [18]. A deletion of the entire atpBEFHAGDC cluster encoding the F1Fo-ATP synthase partially affects cell growth with glucose, and obviously increases pyruvate formation and proton motive force in the amino-acid producing bacterium Corynebacterium glutamicum. The defective process of oxidative phosphorylation in the $\triangle$ F1Fo-ATP synthase mutant leads to a shortage of intracellular ATP, which in turn forces the mutant cells to increase glucose uptake and catabolism for more ATP generation. The results imply that the F1Fo-ATP synthase and oxidative phosphorylation are not utterly indispensable for growth under glucose condition in C. glutamicum [19]. Consistent with these findings, a serineto-proline mutation at residue 237 of $\gamma$ subunit decreased ATP synthase activity to $25 \%$ of wild type in C. glutamicum, but it in turn leads to the enhancement of glucose consumption and respiration rate per cell. The increased glucose catabolism of the $\mathrm{H}^{+}$-ATPase-defective mutant causes an increase in the intracellular pyruvate pool, which is effective for more conversion from pyruvate to L-valine [20]. Moreover, a previous study on the transcriptional analysis of C. glutamicum ATP synthase reveals that the atp operon is expressed optimally at alkaline $\mathrm{pH}$ starting from the upstream of the atpB structural gene, and the sigmaH factor plays an important role in the regulation of the atp operon [21]. Although some studies have focused on the roles of the entire atpBEFHAGDC cluster in C. glutamicum, the physiological functions of two adjacent genes, cg1360 and $\operatorname{cg} 1361$, located just at the upstream of the atp structural genes, had not yet been explored.

In this study, we investigated the potential roles of Cg1360 (encoded by the cg1360 gene) and Cg1361 (encoded by the $c g 1361$ gene) for ATP synthase in C. glutamicum. The data revealed that $\mathrm{Cg} 1360$, but not Cg1361, plays an important role in the maintenance of ATP synthase function and intracellular $\mathrm{NADP}(\mathrm{H})$ levels. Given that NADPH rather than ATP acts as an essential limiting factor for Lvaline formation from the precursor pyruvate, we further examined the potential application of deletion of $\operatorname{cg} 1360$ for efficient L-valine production. Our results supported that Cg1360 can be used as an effective engineering target for the enhancement of L-valine production in industrial biotechnology.

\section{Materials and Methods}

\section{Strains and Growth Conditions}

The plasmids and strains used in this study are listed in Table S1. E. coli DH5 $\alpha$ was used as a host cell for general cloning. C. glutamicum ATCC 13032 was used as the parental strain for gene manipulation and the wild-type (WT) strain for functional analysis. The valine-producing C. glutamicum CGMCC 1.586 was purchased from the China General Microbiological Culture Collection Center (CGMCC, China). A mutant strain V-10, producing a high and stable yield of L-valine, was obtained from C. glutamicum CGMCC 1.586 through multiple rounds of random mutagenesis and screening. Briefly, the original C. glutamicum CGMCC 1.586 strain was treated with $\mathrm{N}$-methyl-N'-nitro-Nnitrosoguanidine (NTG) as a mutagen, and the mutant was selected by flow cytometry measurements based on the Lrp-based amino acid biosensor according to the previous study [22]. The obtained C. glutamicum V-10 strain has been deposited into the China Center of Industrial Culture Collection (CICC, China) under the accession number CICC 24789. E. coli cells were routinely grown at $37^{\circ} \mathrm{C}$ in LB medium $(5 \mathrm{~g} / 1$ yeast extract, $10 \mathrm{~g} / 1$ tryptone, $10 \mathrm{~g} / 1 \mathrm{NaCl}$ ), and $\mathrm{C}$. glutamicum cells were grown at $32^{\circ} \mathrm{C}$ in LBHIS rich medium ( $2.5 \mathrm{~g} / 1$ yeast extract, $5 \mathrm{~g} / 1$ tryptone, $5 \mathrm{~g} / 1 \mathrm{NaCl}, 18.5 \mathrm{~g} / 1 \mathrm{BHI}$ powder and $91 \mathrm{~g} / 1$ sorbitol) or modified CGXII minimal medium (20 g/l (NH4) $\mathrm{SO}_{4}, 5 \mathrm{~g} / 1$ urea, $1 \mathrm{~g} / 1$ $\mathrm{KH}_{2} \mathrm{PO}_{4}, 1 \mathrm{~g} / 1 \mathrm{~K}_{2} \mathrm{HPO}_{4}, 0.25 \mathrm{~g} / 1 \mathrm{MgSO}_{4} \bullet 7 \mathrm{H}_{2} \mathrm{O}, 10 \mathrm{mg} / 1 \mathrm{CaCl}_{2}$ $10 \mathrm{mg} / 1 \mathrm{FeSO}_{4} \bullet 7 \mathrm{H}_{2} \mathrm{O}, 10 \mathrm{mg} / 1 \mathrm{MnSO}_{4} \bullet \mathrm{H}_{2} \mathrm{O}, 1 \mathrm{mg} / 1 \mathrm{ZnSO}_{4} \bullet 7 \mathrm{H}_{2} \mathrm{O}$, $0.2 \mathrm{mg} / 1 \mathrm{CuSO}_{4}, 0.02 \mathrm{mg} / 1 \mathrm{NiCl}_{2} \bullet 6 \mathrm{H}_{2} \mathrm{O}, 0.2 \mathrm{mg} / \mathrm{l}$ biotin, $4 \%$ (w/v) glucose or $100 \mathrm{mM}$ acetic acid) [19]. During the shake-flask fermentation, C. glutamicum strains were cultivated in $250-\mathrm{ml}$ Erlenmeyer flasks containing $30 \mathrm{ml}$ fermentation medium at $32^{\circ} \mathrm{C}$ and $200 \mathrm{rpm}$ for 72 hours. The fermentation medium consisted of $125 \mathrm{~g} / 1$ glucose, $2 \mathrm{~g} / 1$ corn steep liquor, $50 \mathrm{~g} / 1(\mathrm{NH} 4)_{2} \mathrm{SO}_{4}, 1 \mathrm{~g} / 1$ $\mathrm{K}_{2} \mathrm{HPO}_{4}, 0.5 \mathrm{~g} / \mathrm{l} \mathrm{MgSO}{ }_{4} \bullet 7 \mathrm{H}_{2} \mathrm{O}, 100 \mu \mathrm{g} / 1 \mathrm{VB}_{1}, 50 \mu \mathrm{g} / \mathrm{l}$ biotin, $10 \mathrm{~g} / \mathrm{l}$ potassium acetate, $0.2 \mathrm{~g} / 1$ isoleucine, $0.2 \mathrm{~g} / 1$ alanine and $20 \mathrm{~g} / 1$ calcium carbonate. The initial $\mathrm{pH}$ of the above medium was usually adjusted to $\mathrm{pH} 7.0$ and buffered with $200 \mathrm{mM}$ MOPS (3[N-morpholino]-propanesulfonic acid, $\mathrm{pKa}=7.20$ ).

\section{Strain Construction and Complementation}

The primers used in the construction of plasmids and strains 
are listed in Table S2. C. glutamicum mutant strains were achieved by a two-step homologous recombination using the temperaturesensitive plasmid pCRD206 as described previously [23]. In order to avoid polar effects in operon structures, markerless chromosomal in-frame deletion mutants were constructed (Fig. 2A). The $\Delta c g 1360$ mutant contains an in-frame deletion of 127 amino acids of an open reading frame (ORF), while the $\Delta c g 1361$ mutant contains an in-frame deletion of 69 amino acids of an ORF. Chromosomal complementation of the markerless in-frame deletions was also built by a two-step homologous recombination using an approach similar to the above. In order to distinguish the complementation strain $(\Delta c g 1360+c g 1360$ or $\Delta c g 1361+c g 1361)$ with the wild-type strain in genome sequence, a synonymous point mutation at the Glu-4 site (GAA to GAG) of $c g 1360$ gene or at the Leu-4 site (CTG to CTC) of $c g 1361$ gene was introduced (Fig. 2A). The detailed procedures for the construction of the $\Delta c g 1360$, $\Delta c g 1361$ and two complementation strains were provided in the Supplemental Methods.

To detect the spatial distribution of ATP synthase $\beta$ subunit (the product of $a t p D$ gene) in the WT, $\Delta c g 1360$ and $\Delta c g 1361$ strains, the hexa-histidine tag was introduced into its C-terminus according to an approach similar to the above. The L-valine-producing strain C. glutamicum V-10 was used as a starting strain for the fermentative analysis, and the cg1360 gene was disrupted in the $\mathrm{V}-10$ background strain to obtain the V-10- $\Delta$ cg 1360 strain, also according to an approach similar to the above.

\section{Bioinformatics Analysis of AtpI-Like Protein}

Multiple sequence alignment of the putative AtpI from different bacterial species was performed by the Clustal Omega and ESPript 3.0 server [24]. The prediction of transmembrane helices (TMHs) in proteins was performed by the TMHMM Server v. 2.0 [25]. The GenBank accession numbers of the proteins were listed as follows: C. glutamicum ATCC13032 Cg1360, No. CAF19908.1; B. pseudofirmus OF4 ATP synthase protein I, No. ADC49435.1; E. coli K-12 UncI polypeptide, No. AAA24730.1; Pseudomonas putida KT2440 ATP synthase protein I, No. P0A103.1; Shigella boydii Sb227 AtpI, No. ABB68220.1; Clostridium kluyveri DSM 555 AtpI, No. EDK35680.1; Vibrio alginolyticus ATP synthase protein I, No. P12983.1; Pectobacterium atrosepticum SCRI 1043 ATP synthase protein I, No. CAG77414.1.

\section{Growth Assays and Glucose Consumption}

The growth abilities of C. glutamicum WT, mutant and complementation strains were examined in liquid CGXII minimal medium containing $4 \%(\mathrm{w} / \mathrm{v})$ glucose or $100 \mathrm{mM}$ acetic acid as the sole carbon source. Briefly, the corresponding C. glutamicum strains were cultivated for $12 \mathrm{~h}$ at $32^{\circ} \mathrm{C}$ with shaking prior to growth experiments. Overnight cultures were then harvested, washed, and shifted to the CGXII minimal medium containing glucose or acetic acid with an initial optical density at $600 \mathrm{~nm}$ $\left(\mathrm{OD}_{600}\right)$ of 0.1 . Growth curves were established by measuring the $\mathrm{OD}_{600}$ at the indicated time points. The residual glucose concentration in the culture medium was determined by an SBA-40E biosensor analyzer (Institute of Biology, Shandong Academy of Sciences, China). Specific glucose consumption rate during the exponential growth phase was calculated from glucose consumption and biomass concentration using this formula: [decreased glucose $\mathrm{mM}$ ] [increased cell dry weight $(\mathrm{g} / \mathrm{l})]^{-1} \mathrm{~h}^{-1}[26]$. The cell dry weight (CDW) was calculated from the $\mathrm{OD}_{600}$ value using the experimentally correlation factor of $0.25 \mathrm{~g}$ CDW per liter for an $\mathrm{OD}_{600}$ of 1.0 [19].

\section{Determination of the Intracellular ATP and NAD(P)H Concentrations}

C. glutamicum cells grown with glucose as a carbon source were harvested during the exponential growth phase at an $\mathrm{OD}_{600}$ of 3.0 for the determination of intracellular ATP, NADH and NADPH levels. To determine the intracellular ATP concentration, the collected cells were washed once with TE buffer (10 mM Tris-HCl, $1 \mathrm{mM}$ EDTA, pH 7.5). The ATP content was evaluated using a firefly luciferase-based ATP Assay Kit (Beyotime, Shanghai, China) according to the manufacturer's protocols. To determine the intracellular $\mathrm{NAD}(\mathrm{P}) \mathrm{H}$ concentration, the collected cells were washed once with ice-cold phosphate-buffered saline (PBS, $\mathrm{pH}$ 7.5). Next, $300 \mu \mathrm{l}$ of $0.4 \mathrm{M} \mathrm{NaOH}$ was added to the cells for homogenization following a water bath for $10 \mathrm{~min}$ at $50^{\circ} \mathrm{C}$. Then, $300 \mu \mathrm{l}$ of $0.4 \mathrm{M} \mathrm{HCl}$ was slowly added to neutralize the extract, and the mixture was shaken mildly and centrifuged at $8,000 \times g$ for $5 \mathrm{~min}$ [27]. The levels of NADH or NADPH in extracts were quantified using a spectrophotometric enzyme cycle method as described previously $[28,29]$. The reaction mixture for NADH determination consisted of $100 \mathrm{mM}$ Bicine buffer ( $\mathrm{pH} 8.0), 4 \mathrm{mM}$ EDTA (pH 8.0), $3.32 \mathrm{mM}$ phenazine ethyl sulfate, $0.42 \mathrm{mM}$ thiazolyl blue, $5 \mathrm{U} / \mathrm{ml}$ alcohol dehydrogenase (ADH), 10\% (v/v) ethanol and appropriate volume of cell extract. The reaction mixture for NADPH determination was the same as the NADH assay mixture except that $\mathrm{ADH}$ and ethanol were replaced with $1.75 \mathrm{U} / \mathrm{ml}$ glucose-6-phosphate dehydrogenase (G6PDH) and 3.8 $\mathrm{mM}$ glucose-6-phosphate. All the experiments were performed in triplicate and repeated three times with similar results.

\section{Real-Time Quantitative PCR Analysis}

Quantitative real-time reverse transcription-PCR (qPCR) was used to calculate the expression levels of the target genes. The primers used in this experiment are listed in Table S2. RNA extraction and reverse transcription were performed using a Bacterial Total RNA Extraction Kit (Tiangen Biotech, China) and a Revert Aid First Strand cDNA Synthesis Kit (Thermo Scientific, USA). For relative qPCR analysis, the reaction mixture was prepared using SYBR Green Real-Time PCR Master Mix (Toyobo, Japan) with reference to the instructions, while data acquisition and analysis were realized using an Applied Biosystems 7500 Fast Real-Time PCR System. The 16S rRNA gene (encoding for RNA component of bacterial 30S ribosomal subunit) and a commonly used housekeeping gyrB gene (encoding for the B subunit of DNA gyrase) have been previously used as candidate reference genes 
for qPCR analysis. The two genes are proved to be a relatively stable set of reference genes in many bacterial species, including C. glutamicum [30-32]. Similar experimental results were obtained according to these two reference genes in this study, and the $16 \mathrm{~S}$ rRNA was used as a normalized reference gene for the present data. The relative fold changes in gene expression were calculated according to the delta delta threshold cycle method as described previously [33].

Assays for ATPase Hydrolysis Activity, ATP-Driven Proton Pumping Activity, and the Spatial Distribution of $\beta$ Subunit

C. glutamicum cells grown with glucose as a carbon source were harvested during the exponential growth phase at an $\mathrm{OD}_{600}$ of 3.0, and washed once with ice-cold PBS buffer. Then, cell pellets were resuspended in $35 \mathrm{ml}$ of PS4 buffer ( $50 \mathrm{mM}$ Tris- $\mathrm{HCl}, \mathrm{pH} 7.5$, $10 \mathrm{mM} \mathrm{MgCl}{ }_{2}, 10 \%$ (v/v) glycerol, $1 \mathrm{mM}$ phenylmethylsulfonyl fluoride, $0.3 \mathrm{mg} / \mathrm{ml}$ DNase I, one complete protease inhibitor tablet per $50 \mathrm{ml}$ buffer). Inverted membrane vesicles were prepared as described previously $[9,34]$. In brief, the cells were disrupted using a French press (15,000 p.s.i) and the crushed suspension was centrifuged at $15,000 \times g$ for $15 \mathrm{~min}$ to remove cell debris and unbroken cells. The supernatant was centrifuged at 200,000 $\times g$ in an SW70 Ti Rotor, Optima LE-80K Ultracentrifuge (Beckman Coulter, USA) for $1 \mathrm{~h}$ at $4^{\circ} \mathrm{C}$. The pellets were suspended in TCDG buffer (10 mM Tris- $\mathrm{HCl}, \mathrm{pH} 7.5,140 \mathrm{mM}$ choline chloride, $0.5 \mathrm{mM}$ dithiothreitol; $10 \%$ glycerol) using homogenizer, and stored at $-80^{\circ} \mathrm{C}$ before use. The determination of ATPase hydrolysis activity was performed by measuring the amount of released inorganic phosphate from ATP [35]. The determination of ATP-driven proton-pumping activity was determined by the measurement of 9-amino-6-chloro-2methoxyacridine (ACMA) fluorescence quenching [9]. All the experiments were performed in triplicate and repeated at least three times.

To assess the total amount of the $\beta$ subunit and its distribution between the cytoplasm and the membrane, the total protein, as well as the cytoplasmic fraction and membrane fraction were collected. Protein concentration was measured by the Bradford method (Bio-Rad, USA). Equal amounts of sample protein derived from different strains were loaded separately onto the sodium dodecyl sulfate polyacrylamide gel electrophoresis (SDS-PAGE) gels, and examined by western blotting analysis using Histag antibody. Protein bands were quantified using the ImageJ gel analysis software (Wayne Rasband, NIH).

\section{Results and Discussion}

\section{Sequence Analyses of Cg1360 and Cg1361}

Most bacterial ATP operons have 8 structural genes in the order atp BEFHGADC [8], and a ninth gene, named atpI gene, is located at the upstream of the atp operon (Fig. 1A). We first analyzed the complete genome sequence of
C. glutamicum ATCC 13032 [36]. Two unknown ORFs, with locus tags of cg1360 and cg1361, were located at the upstream of the atp operon in C. glutamicum (Fig. 1A). The alignments of Cg1360 with some related AtpI protein were shown in Figs. 1B and S1. The cg1360 gene was predicted to encode a hydrophobic protein with $4 \mathrm{TMHs}$, while no TMH was found in Cg1361. The AtpI protein from other microorganisms, such as E. coli, and B. pseudofirmus OF4, has also been predicted to have 4 TMHs, suggesting that Cg1360, rather than Cg1361, in C. glutamicum may play a role as AtpI in the atp operon. However, Cg1360 showed low sequence identity to the predicted AtpI from E. coli ( $23 \%$ identity, $34 / 149$ aa), and to the AtpI from $B$. pseudofirmus OF4 (16\% identity, 24/149 aa) (Table S3). Similarly, no apparent sequence homology was found for Cg1361 (Table S4). These findings indicated that the sequence of Cg1360/Cg1361 shares no obvious homology with the putative AtpI from different organisms.

\section{Effects of Deletion of $\operatorname{cg} 1360$ or $\operatorname{cg} 1361$ on Cell Growth and Glucose Consumption}

The $\Delta \operatorname{cg} 1360, \Delta c g 1361$ and two related complementation strains were successfully constructed as described in Materials and Methods. The growth properties of the WT, $\Delta c g 1360$ and $\Delta c g 1361$ strains were compared under the conditions of different carbon sources (Fig. 2, Table 1). The $\Delta c g 1360$ strain exhibited a large growth defect relative to the WT control (around 70\% of WT) when glucose was used as the sole carbon source (Fig. 2B). In prokaryotic microorganisms, the metabolic utilization of acetate to produce acetyl-CoA requires the use of ATP. In the presence of acetate as a sole carbon source, most ATP is generated by oxidative phosphorylation while substratelevel phosphorylation provides a less efficient source of ATP $[19,37]$. Here, we found that the $\Delta c g 1360$ strain exhibited a more severe growth defect in the acetate-containing medium (Fig. 2C), which had only 31\% biomass of the WT control (Table 1). As mentioned earlier, Cg1360 may belong to the homologous protein of bacterial AtpI, so we speculate that loss of cg1360 may cause a deficiency of ATP synthase function, resulting in an insufficient ATP supply for acetate utilization. However, deletion of $c g 1361$ had no obvious impact on cell growth in either glucose or acetate carbon sources (Figs. 2B and 2C). In addition, there was no statistically significant difference in the growth parameters among the WT and two complementation strains (Fig. 2 and Table 1), indicating that the changes observed in the $\Delta c g 1360$ mutant were indeed caused by gene deletion at the chromosomal level. Interestingly, the glucose consumption 
A Escherichia coli K-12

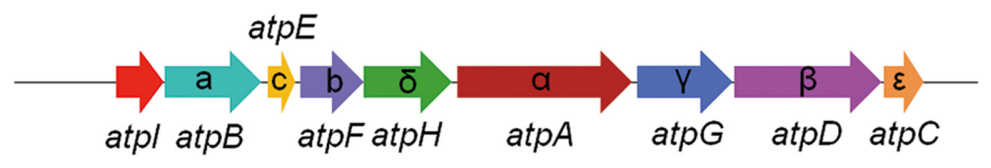

Bacillus pseudofirmus OF4

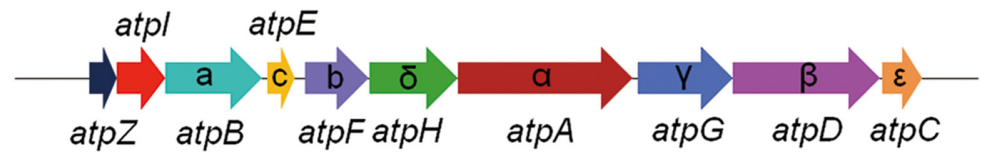

Corynebacterium glutamicum ATCC 13032

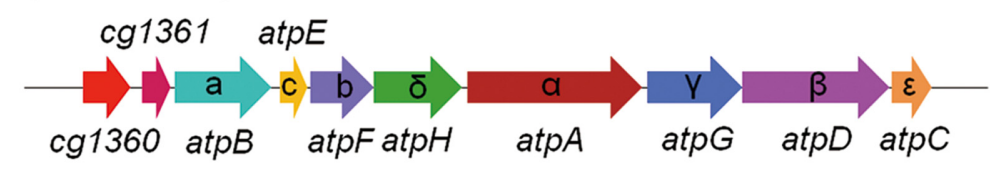
B C. glutamicum
B. pseudo
E. coli
P. putida
S. boydii
V. alginolyticus
$P$. atrosepticum
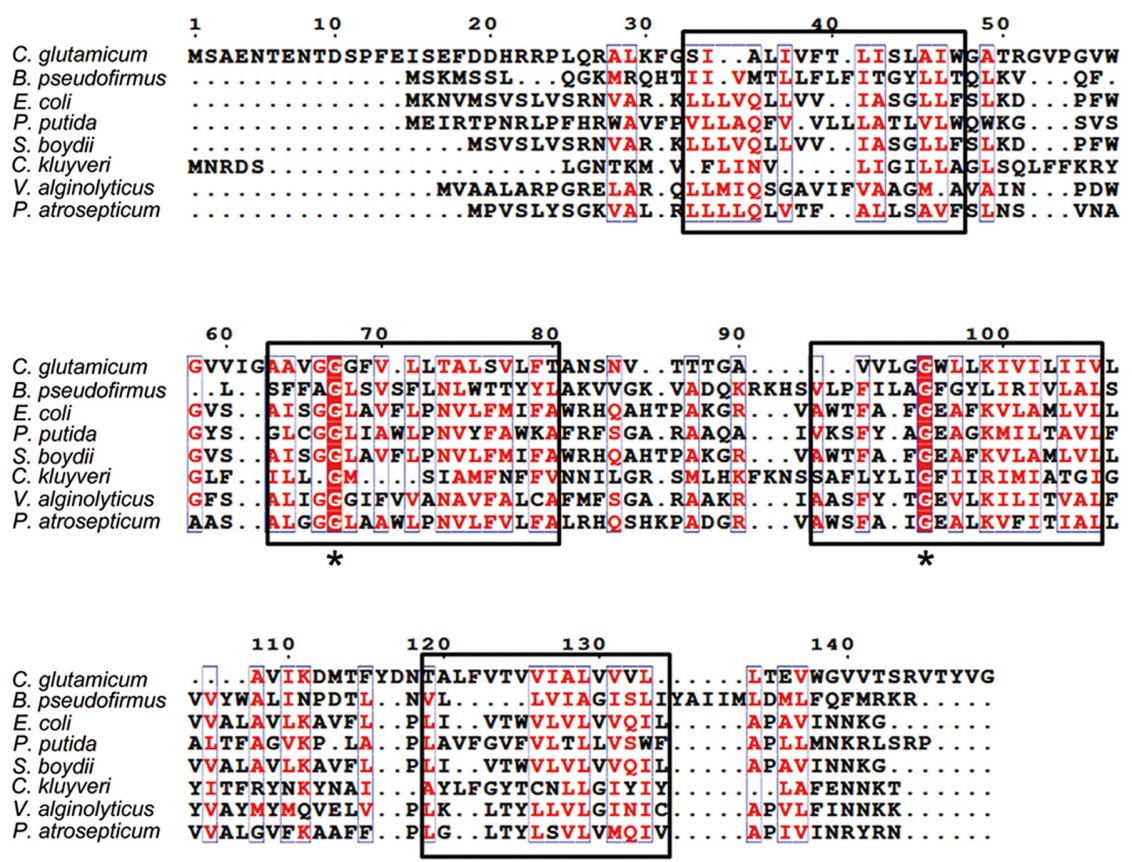

Fig. 1. Bioinformatics analysis of bacterial AtpI-like protein.

(A) Gene arrangements of the atp operon in E. coli K-12, B. pseudofirmus OF4 and C. glutamicum ATCC 13032. (B) Multiple sequence alignment of the putative AtpI from different bacterial species. The sequence alignment was performed by the Clustal Omega and ESPript 3.0 server. The prediction of transmembrane helices in proteins was carried out by the TMHMM Server, and shown as black boxes.

rate of the $\Delta c g 1360$ mutant during the exponential growth phase indeed showed 39\% higher than the WT control (Fig. S2A). The enhancement of the glucose consumption rate by defective F1Fo-ATP synthase has also been reported for E. coli [16, 38], Torulopsis glabrata [39] and C. glutamicum [40], indicating a strategy enabling microorganisms to survive in energy shortages caused by the ATP synthase defect.

Roles of $\operatorname{cg} 1360$ or $\operatorname{cg} 1361$ on the Assembly of ATP Synthase, ATP-Driven Proton-Pumping Activity and ATPase Activity In order to confirm whether cg1360 and cg1361 were 

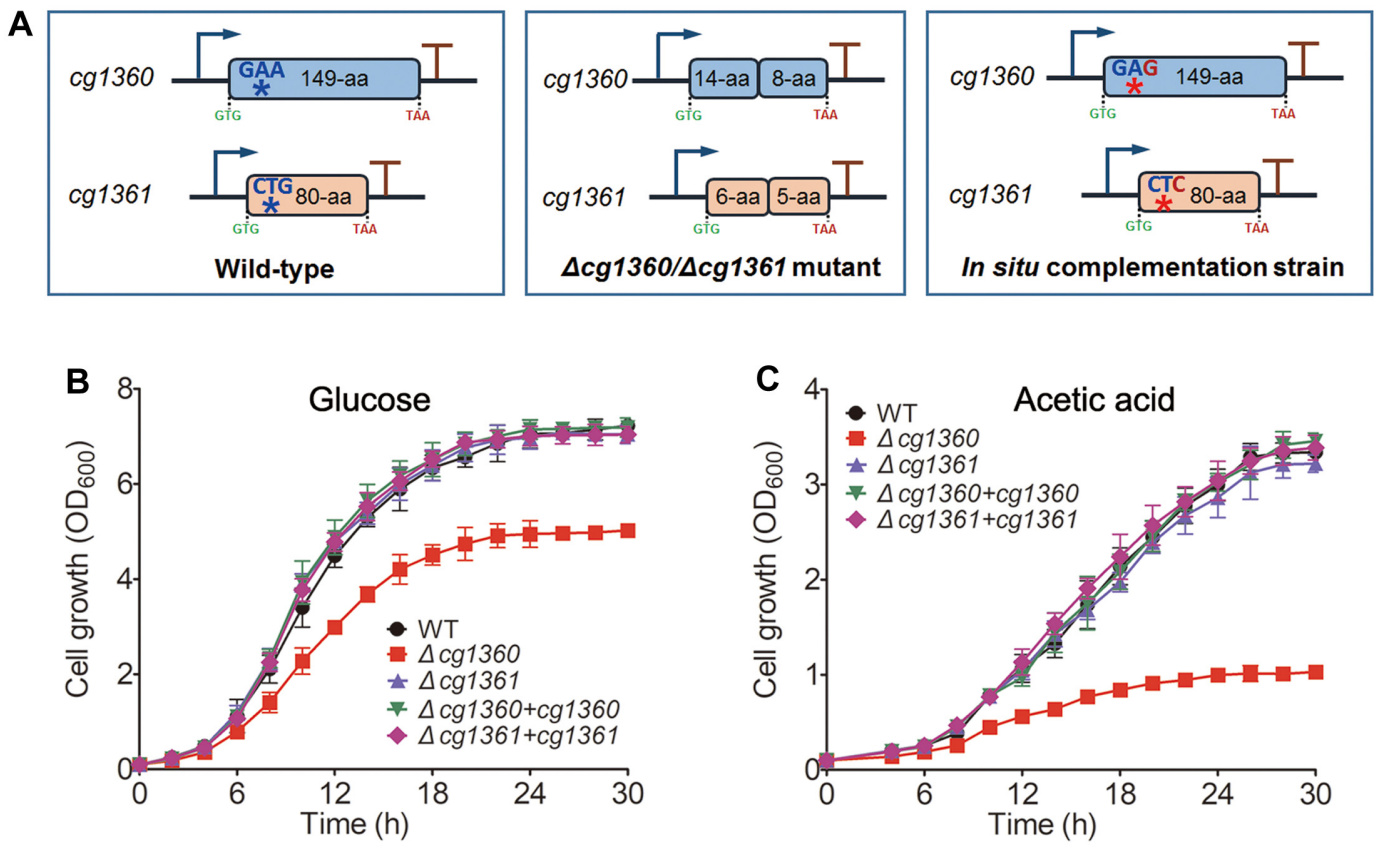

Fig. 2. Growth assays of the wild-type, $\Delta \operatorname{cg} 1360, \Delta c g 1361$, and two complementation strains.

(A) Sequence differences at the chromosomal locus of $c g 1360$ or $c g 1361$ gene among wild-type, $\Delta c g 1360, \Delta c g 1361$ and two complementation strains. * Indicates the synonymous point mutation in genome. (B and C) Growth abilities were examined in CGXII minimal medium containing $4 \%$ (w/v) glucose or $100 \mathrm{mM}$ acetic acid as sole carbon sources. Overnight cultures were resuspended in the liquid medium with an initial $\mathrm{OD}_{600}$ of 0.1 , and growth curves were established by measuring the $\mathrm{OD}_{600}$ at the indicated time points.

associated with the expression of the ATP synthase operon, the transcriptional levels of ATP synthase components were measured by qPCR. Given that the ATP synthase operon contains 8 different adjacent genes, we chose four representative genes $(\operatorname{atp} B$, atpE, $\operatorname{atpH}$, and $\operatorname{atpD})$ to evaluate the transcription pattern of the whole operon. As shown in Fig. 3A, the transcription levels of atpB, atpE, atpH, and atpD in the $\Delta c g 1360$ mutant were at least $55 \%$ lower than those of the WT control, indicating that cg1360 may play an important role in the transcriptional activation of the ATP synthase operon. However, lack of the cg1361 gene had no significant influence on the expression of the ATP synthase operon.

AtpI from P. modestum is indispensable for the production of active ATP synthase when expressing a hybrid F1Fo in E. coli cells [12]. Functional c-rings can only be produced in the presence of both AtpI and c subunits [11]. Additionally, our previous study has shown that AtpI from $B$.

Table 1. Cell growth, $\beta$ subunit distribution, ATPase activity and ATP-driven proton-pumping activity assays for C. glutamicum wild-type strain and its mutant derivatives.

\begin{tabular}{|c|c|c|c|c|c|c|c|c|}
\hline \multirow[t]{2}{*}{ Strain } & \multicolumn{2}{|c|}{$\begin{array}{l}\text { Glucose } \\
(24 \mathrm{~h})\end{array}$} & \multicolumn{2}{|c|}{$\begin{array}{c}\text { Acetic acid } \\
(30 \mathrm{~h})\end{array}$} & \multicolumn{2}{|c|}{$\begin{array}{c}\beta \text { subunit content } \\
(\% \text { of } \mathrm{WT})\end{array}$} & \multirow{2}{*}{$\begin{array}{c}\text { ATPase } \\
\text { activity } \\
(\% \text { of WT })\end{array}$} & \multirow{2}{*}{$\begin{array}{c}\text { Proton-pumping } \\
\text { activity } \\
(\% \text { of WT) }\end{array}$} \\
\hline & Growth $\left(\mathrm{OD}_{600}\right)$ & $\%$ of $\mathrm{WT}^{\mathrm{a}}$ & Growth $\left(\mathrm{OD}_{600}\right)$ & $\%$ of WT & Membrane & Cytoplasm & & \\
\hline WT & $7.1 \pm 0.3$ & 100 & $3.2 \pm 0.06$ & 100 & 100 & 100 & 100 & 100 \\
\hline$\Delta c g 1360$ & $5.0 \pm 0.3$ & 70 & $1.0 \pm 0.03$ & 31 & $66 \pm 5$ & $235 \pm 15$ & $78 \pm 4$ & $55 \pm 6$ \\
\hline$\Delta c g 1361$ & $7.0 \pm 0.2$ & 99 & $3.1 \pm 0.09$ & 96 & $97 \pm 6$ & $104 \pm 9$ & $99 \pm 5$ & $96 \pm 4$ \\
\hline$\Delta c g 1360+\operatorname{cg} 1360$ & $7.2 \pm 0.2$ & 101 & $3.3 \pm 0.08$ & 103 & $\mathrm{ND}^{\mathrm{b}}$ & ND & ND & ND \\
\hline$\Delta c g 1361+\operatorname{cg} 1361$ & $7.0 \pm 0.4$ & 99 & $3.2 \pm 0.10$ & 100 & $\mathrm{ND}$ & ND & ND & ND \\
\hline
\end{tabular}

${ }^{a}$ The percentage (\%) was calculated based on $\mathrm{OD}_{600}, \beta$ subunit content, ATPase activity or proton-pumping activity of recombinant strains compared to that of wild-type control, respectively.

${ }^{\mathrm{b}} \mathrm{ND}$ represents not detected. 

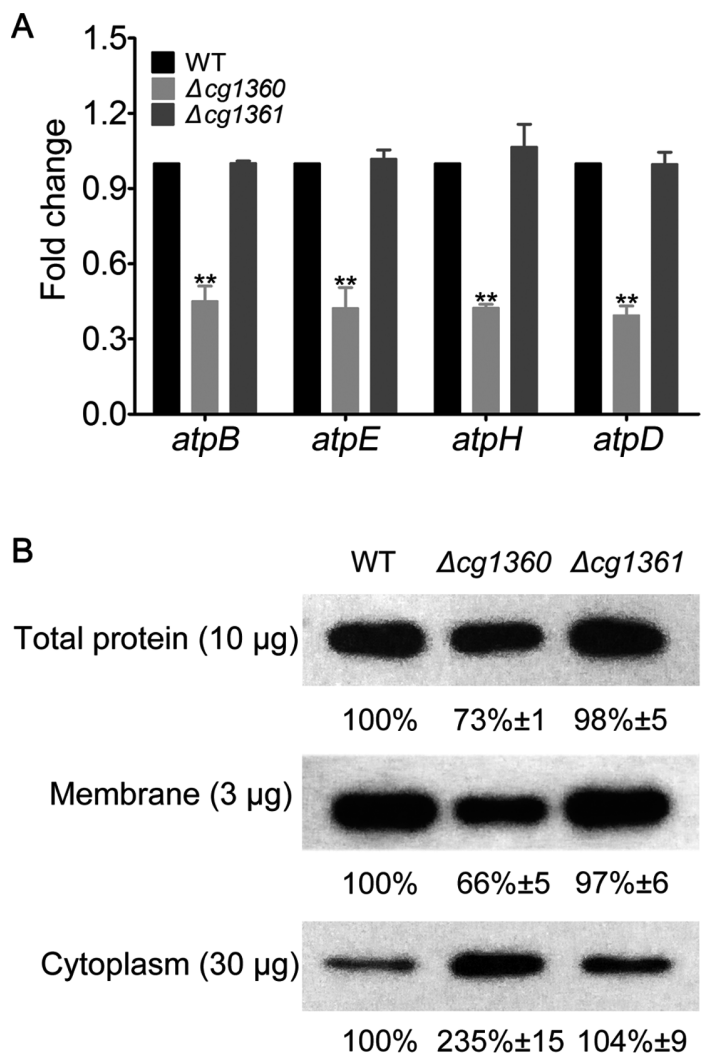

Fig. 3. Effects of $\operatorname{cg} 1360$ or $\operatorname{cg} 1361$ deletion on the expression and assembly of the ATP synthase.

(A) Transcription levels of genes encoding ATP synthase subunits were quantified using real-time qPCR and fold changes were calculated relative to the wild-type levels (assigned a value of 1 ). Asterisks indicate significant differences between C. glutamicum wild type and its mutant derivatives according to a statistical Student's $t$-test $\left(^{* *}, p<0.01\right)$. (B) The total protein levels and spatial distribution of $\beta$ subunit of ATP synthase in the wild type and its mutant derivatives. Image analysis was performed using the ImageJ software for quantitative purposes and the wild-type content was arbitrarily designated as $100 \%$.

pseudofirmus OF4 is not absolutely required for c-ring assembly, but deletion of the atpI gene decreases the c-ring stability [9]. Therefore, we further investigated the effects of deletion of $c g 1360$ or cg1361 in C. glutamicum on the assembly of ATP synthase by introducing a $6 \times$ His tag into $\beta$ subunit of ATP synthase F1 sector. If the peripheral F1 portions cannot properly be assembled with the hydrophobic Fo component to form an intact ATP synthase at the membrane surface, the increased levels of F1 ( $\beta$ subunit) will be detected in the cytoplasm. As shown in Fig. 3B, the total expression levels of ATP synthase $\beta$ subunit in the $\Delta c g 1360$ mutant was only $73 \%$ of the WT level, consistent with the evidence of the reduced transcriptional levels of atp operon. Deletion of $c g 1360$ gene resulted in a $34 \%$ reduction, relative to the $\mathrm{WT}$, in the content of ATP synthase $\beta$ subunit in the membrane fraction, and a $235 \%$ increase of $\beta$ subunit content was detected in the cytoplasm compared to that in the WT (Table 1). It should be noted that deletion of cg1360 did not completely abolish the appearance of $\beta$ subunit on cell membrane, indicating that cg1360 can affect the assembly of intact ATP synthase, but it may not be an indispensable factor. In addition, deletion of $c g 1361$ had no obvious effects on the total protein levels of $\beta$ subunit and its distribution between membrane and the cytoplasm. Taken together, the above findings suggested that Cg1360 plays important roles in controlling the expression and assembly of ATP synthase in C. glutamicum, which is inconsistent with the putative role of AtpI from other microorganisms [9, 12].

The effects of deletion of $c g 1360$ and cg1361 on ATPdriven proton-pumping activity and ATPase hydrolysis activity were also investigated (Fig. 4 and Table 1). Deletion of cg1360 resulted in a $45 \%$ decrease in protonpumping activity compared to that in the $\mathrm{WT}$, whereas deletion of cg1361 slightly lowered the proton-pumping activity of ATP synthase in C. glutamicum. The ATPase hydrolysis activity of the $\Delta c g 1360$ mutant only accounted for $78 \%$ of the WT, while the $\Delta c g 1361$ mutant showed no obvious ATPase deficiency. The decreased proton-pumping activity and ATPase activity in the $\Delta c g 1360$ mutant may be partly attributed to the findings that the absence of cg1360 had a negative effect on the expression and assembly of ATP synthase complexes.

\section{Comparison Analysis of the Intracellular ATP and NAD(P)H Concentrations}

The decreased activity of ATP synthase, including ATPdriven proton-pumping activity and ATPase activity, in the $\Delta c g 1360$ mutant prompted us to further analyze the levels of intracellular ATP and NAD(P)H. As shown in Fig. 5, deletion of $c g 1360$ resulted in a $28 \%$ decrease in the intracellular ATP concentrations, relative to the WT level. However, the levels of intracellular NADH and NADPH in the $\Delta c g 1360$ mutant increased by $29 \%$ and $26 \%$, respectively. As expected, re-introducing the native cg1360 gene into the $\Delta c g 1360$ mutant could restore the levels of intracellular ATP and NAD(P)H back to the WT level. The decreased ATP accumulation was in agreement with the growth phenotypes of the $\Delta c g 1360$ mutant that exhibited an impaired growth under glucose condition and a more severe growth defect with acetate as the sole carbon source 
A

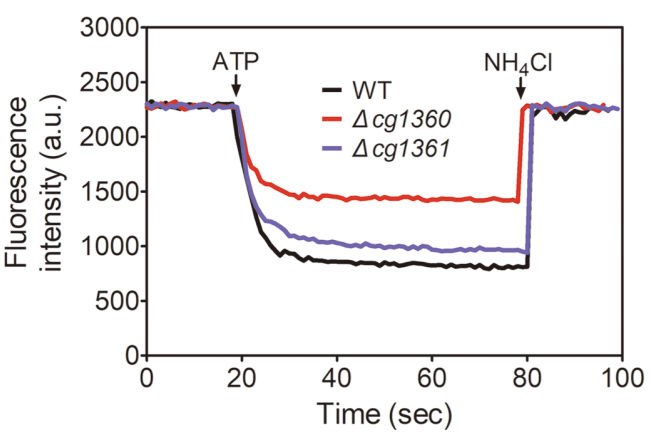

B

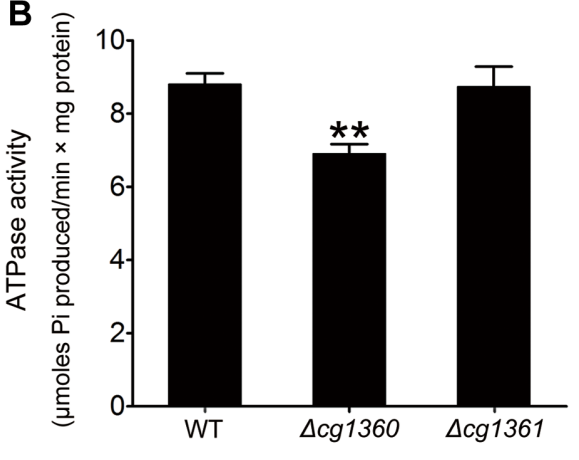

Fig. 4. Effects of $c g 1360$ or $c g 1361$ deletion on the ATP-driven pumping and ATPase hydrolysis activities.

(A) ATP-driven proton-pumping activities in membrane vesicles from wild type and its mutant derivatives were monitored by fluorescence quenching of the $\mathrm{pH}$-sensitive probe $\mathrm{ACMA}$. $\mathrm{NH}_{4} \mathrm{Cl}$ was added to the assay buffer to dissipate the proton gradient and establish a baseline. (B) ATPase hydrolytic activities of wild type and its mutant derivatives were evaluated by measuring the amount of inorganic phosphate produced by ATP substrate.

(Fig. 2), supporting the importance of ATP supply to cell growth. Moreover, the $\Delta c g 1361$ mutant exhibited comparable levels of the intracellular ATP and $\mathrm{NAD}(\mathrm{P}) \mathrm{H}$ with the WT strain, suggesting its minor role in the regulation of ATP synthase.

In general, ATP synthase synthesizes ATP by a rotary mechanism driven by a transmembrane proton motive force (pmf) produced by the respiratory chain [6, 41, 42]. The NADH, as the electron donor, can transfer electrons to the respiratory chain, and is considered as the major donor to create the proton gradient for ATP synthesis. Therefore, it was reasonable that the deficiency of ATP production caused by deletion of $c g 1360$ would lead to the accumulation of intracellular reducing equivalents.
Enhancement of L-Valine Production with Deletion of cg1360 in C. glutamicum

C. glutamicum is regarded as a generally-recognized-assafe (GRAS) bacterium, and has been successfully engineered as an important platform for the production of various amino acids [43-45]. L-valine is synthesized from pyruvate without the requirement of ATP via the four-step enzymatic steps, and NADPH acts as a limiting cofactor in the second step (Fig. 6A) [20,46]. In view of the above findings that deletion of $c g 1360$ led to a significant increase in the $\mathrm{NAD}(\mathrm{P}) \mathrm{H}$ levels, we further investigated the effect of deletion of $\operatorname{cg} 1360$ on the production of L-valine. As shown in Fig. 6B, deletion of cg1360 in the L-valine-producing strain of V-10 resulted in a $23 \%$ decrease in cell growth
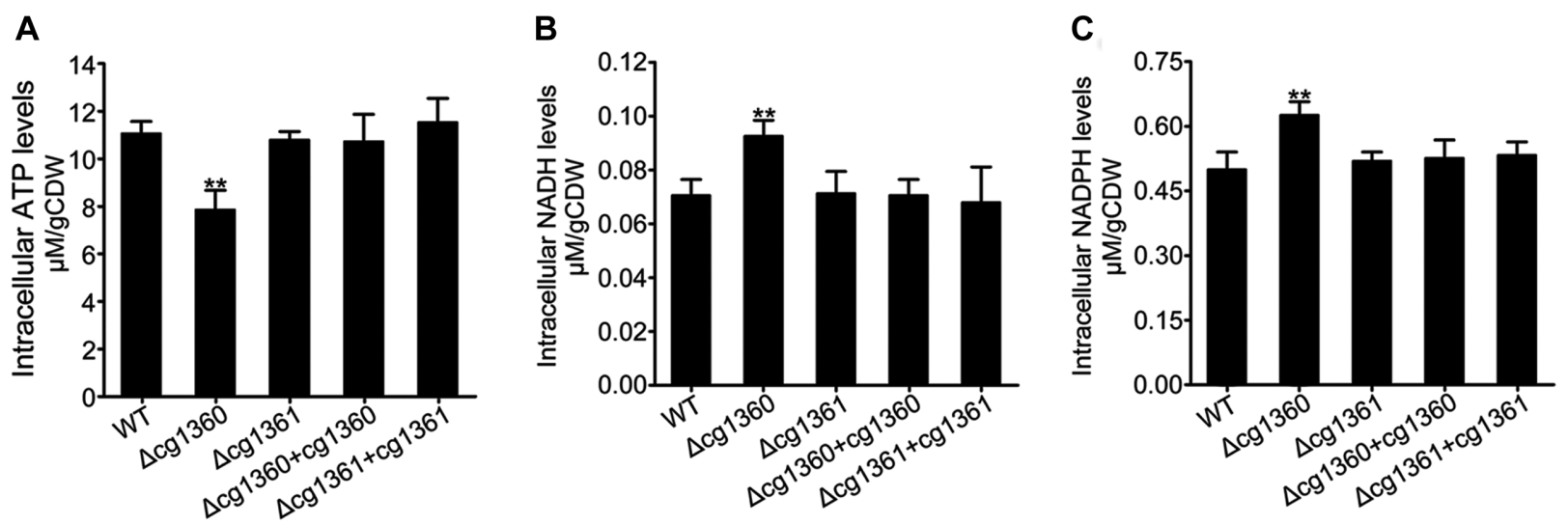

Fig. 5. Effects of $c g 1360$ or $c g 1361$ deletion on the intracellular ATP (A), NADH (B), and NADPH (C) levels.

The ATP content was measured using a firefly luciferase-based ATP assay kit, and the NAD(P)H content was quantified using a spectrophotometric enzymatic cycling method as described previously. 
A
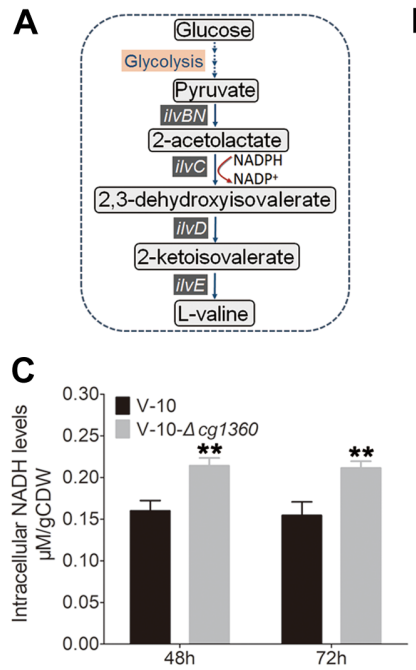

E

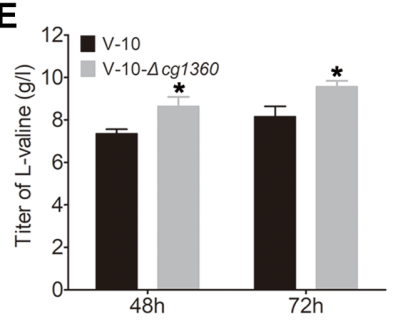

B

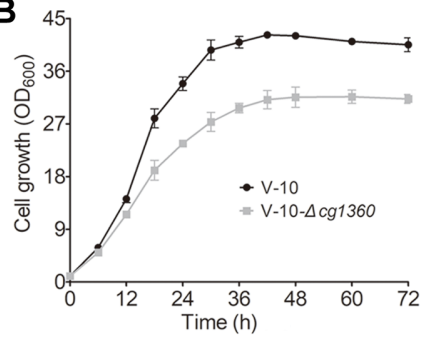

D

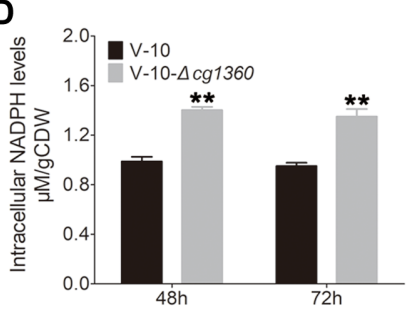

$\mathbf{F}$

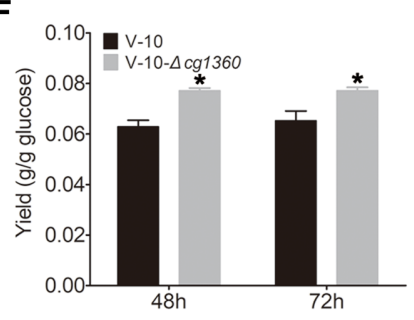

Fig. 6. Effects of $c g 1360$ deletion on L-valine production in shake flask fermentation.

(A) The biosynthetic pathway of L-valine in C. glutamicum. (B) Growth curves of L-valine-producing C. glutamicum V-10 strain and its V-10 mutant lacking cg1360 in shake flask cultures. (C and D) The intracellular NADH and NADPH levels after $48 \mathrm{~h}$ or $72 \mathrm{~h}$ of fermentation of V-10 and V-10-4cg1360 strains. (E and F) The total Lvaline titer and yield after $48 \mathrm{~h}$ or $72 \mathrm{~h}$ of fermentation of V-10 and V10- $\Delta c g 1360$ strains. Asterisks indicate significant differences between C. glutamicum $\mathrm{V}-10$ and its mutant according to a statistical Student's $\mathrm{t}$ test $\left(^{*}, p<0.05\right)$.

compared to that in V-10 strain, which was similar to the observations of the $\Delta c g 1360$ mutant in the wild-type background (Fig. 2B). The glucose consumption rate of the $\mathrm{V}-10-\Delta c g 1360$ mutant during the exponential growth phase was $28 \%$ higher than that of the V-10 strain, implying that the mutant has a stronger carbon metabolism (Fig. S2B). As expected, the levels of intracellular NADH and NADPH in the V-10- $\Delta c g 1360$ mutant increased by $40 \%$ and $42 \%$ after $72 \mathrm{~h}$ of fermentation as compared to the V-10 control (Figs. 6C and 6D), whereas deletion of $c g 1360$ in the V-10 background strain resulted in an approximately $24 \%$ decrease in intracellular ATP concentrations (Fig. S3). The final production of L-valine in the V-10- $\Delta \operatorname{cg} 1360$ mutant reached $9.2 \pm 0.3 \mathrm{~g} / 1$ with a yield of $0.078 \pm 0.001 \mathrm{~g} / \mathrm{g}$

glucose after $72 \mathrm{~h}$ of fermentation in shake flasks, which was $14 \%$ higher than that of the starting V-10 strain (Figs. 6E and 6F). The improved L-valine titer of the V-10$\Delta c g 1360$ mutant could be attributed to an overall increase of the intracellular reducing equivalents. The effectiveness of the ATP synthase defects for the production of useful metabolites, such as pyruvate and amino acids, has also been reported for E. coli and C. glutamicum [16, 20, 40]. A previous study reveals that the combination of an $\mathrm{H}^{+}$ATPase defect with the feedback-insensitive acetohydroxyacid synthase (ilvBN products) could enhance L-valine production from $2.54 \mathrm{~g} / 1$ to $5.47 \mathrm{~g} / 1$ in the shaking flasks [20]. Consistent with these above characterizations, our results showed the enhancement of L-valine production by deleting the cg1360 gene in atp operon of C. glutamicum, suggesting that $\operatorname{cg} 1360 \mathrm{can}$ be expected to offer a promising alternative candidate from the perspective of energy regulation for strain improvement. This energy deficiency strategy can potentially be applied for the fermentative production of other useful chemicals.

\section{Acknowledgments}

This study was supported by the Natural Science Foundation of Tianjin City (No.17JCYBJC24000), the National Key Research and Development Program of China (No. 2018YFA0901002), the National Natural Science Foundation of China (No. 31801526, No. 31601460), and the Tianjin Science and Technology Project (No.15PTCYSY00020, No.14ZCZDSY00157).

\section{Conflict of Interest}

The authors have no financial conflicts of interest to declare.

\section{References}

1. Nakamoto RK, Baylis Scanlon JA, Al-Shawi MK. 2008. The rotary mechanism of the ATP synthase. Arch. Biochem. Biophys. 476: 43-50.

2. von Ballmoos C, Wiedenmann A, Dimroth P. 2009. Essentials for ATP synthesis by F1Fo-ATP synthases. Annu. Rev. Biochem. 78: 649-672.

3. Hicks DB, Liu J, Fujisawa M, Krulwich TA. 2010. F1Fo-ATP synthases of alkaliphilic bacteria: lessons from their adaptations. Biochim. Biophys. Acta 1797: 1362-1377.

4. Kulish O, Wright AD, Terentjev EM. 2016. F1 rotary motor of ATP synthase is driven by the torsionally-asymmetric drive shaft. Sci. Rep. 6: 28180. 
5. Nesci S, Trombetti F, Ventrella V, Pagliarani A. 2015. Opposite rotation directions in the synthesis and hydrolysis of ATP by the ATP synthase: hints from a subunit asymmetry. J. Membr. Biol. 248: 163-169.

6. Capaldi RA, Aggeler R. 2002. Mechanism of the F1Fo-type ATP synthase, a biological rotary motor. Trends Biochem. Sci. 27: 154-160.

7. Neupane P, Bhuju S, Thapa N, Bhattarai HK. 2019. ATP Synthase: structure, function and inhibition. Biomol. Concepts 10: $1-10$.

8. Gay NJ, Walker JE. 1981. The atp operon: nucleotide sequence of the region encoding the alpha-subunit of Escherichia coli ATP-synthase. Nucleic Acids Res. 9: 2187-2194.

9. Liu J, Hicks DB, Krulwich TA. 2013. Roles of AtpI and two YidC-type proteins from alkaliphilic Bacillus pseudofirmus OF4 in ATP synthase assembly and nonfermentative growth. J. Bacteriol. 195: 220-230.

10. Gay NJ. 1984. Construction and characterization of an Escherichia coli strain with a uncI mutation. J. Bacteriol. 158: 820-825.

11. Suzuki T, Ozaki Y, Sone N, Feniouk BA, Yoshida M. 2007. The product of uncI gene in F1Fo-ATP synthase operon plays a chaperone-like role to assist c-ring assembly. Proc. Natl. Acad. Sci. USA 104: 20776-20781.

12. Ozaki Y, Suzuki T, Kuruma Y, Ueda T, Yoshida M. 2008. UncI protein can mediate ring-assembly of c-subunits of F1Fo-ATP synthase in vitro. Biochem. Biophys. Res. Commun. 367: 663-666.

13. Koebmann BJ, Westerhoff HV, Snoep JL, Nilsson D, Jensen PR. 2002. The glycolytic flux in Escherichia coli is controlled by the demand for ATP. J. Bacteriol. 184: 3909-3916.

14. Hara KY, Kondo A. 2015. ATP regulation in bioproduction. Microb. Cell Fact. 14: 198.

15. Zhou J, Liu L, Shi Z, Du G, Chen J. 2009. ATP in current biotechnology: regulation, applications and perspectives. Biotechnol. Adv. 27: 94-101.

16. Causey TB, Shanmugam KT, Yomano LP, Ingram LO. 2004. Engineering Escherichia coli for efficient conversion of glucose to pyruvate. Proc. Natl. Acad. Sci. USA 101: 2235-2240.

17. Causey TB, Zhou S, Shanmugam KT, Ingram LO. 2003. Engineering the metabolism of Escherichia coli W3110 for the conversion of sugar to redox-neutral and oxidized products: homoacetate production. Proc. Natl. Acad. Sci. USA 100: 825832.

18. Wada M, Narita K, Yokota A. 2007. Alanine production in an $\mathrm{H}^{+}$-ATPase- and lactate dehydrogenase-defective mutant of Escherichia coli expressing alanine dehydrogenase. Appl. Microbiol. Biotechnol. 76: 819-825.

19. Koch-Koerfges A, Kabus A, Ochrombel I, Marin K, Bott M. 2012. Physiology and global gene expression of a Corynebacterium glutamicum $\triangle \mathrm{F} 1 \mathrm{Fo}$-ATP synthase mutant devoid of oxidative phosphorylation. Biochim. Biophys. Acta 1817: 370380 .
20. Wada M, Hijikata N, Aoki R, Takesue N, Yokota A. 2008. Enhanced valine production in Corynebacterium glutamicum with defective $\mathrm{H}^{+}$-ATPase and C-terminal truncated acetohydroxyacid synthase. Biosci. Biotechnol. Biochem. 72: 29592965.

21. Barriuso-Iglesias M, Barreiro C, Sola-Landa A, Martin JF. 2013. Transcriptional control of the F1Fo-ATP synthase operon of Corynebacterium glutamicum: SigmaH factor binds to its promoter and regulates its expression at different $\mathrm{pH}$ values. Microb. Biotechnol. 6: 178-188.

22. Mustafi N, Grünberger A, Kohlheyer D, Bott M, Frunzke J. 2012. The development and application of a single-cell biosensor for the detection of 1-methionine and branchedchain amino acids. Metab. Eng. 14: 449-457.

23. Okibe N, Suzuki N, Inui M, Yukawa H. 2011. Efficient markerless gene replacement in Corynebacterium glutamicum using a new temperature-sensitive plasmid. J. Microbiol. Methods 85: 155-163.

24. Robert X, Gouet P. 2014. Deciphering key features in protein structures with the new ENDscript server. Nucleic Acids Res. 42: W320-324.

25. Tusnady GE, Simon I. 2010. Topology prediction of helical transmembrane proteins: how far have we reached? Curr. Protein Pept. Sci. 11: 550-561.

26. Sawada K, Kato Y, Imai K, Li L, Wada M, Matsushita K, et al. 2012. Mechanism of increased respiration in an $\mathrm{H}^{+}-$ ATPase-defective mutant of Corynebacterium glutamicum. J. Biosci. Bioeng. 113: 467-473.

27. Lee IY, Kim MK, Park YH, Lee SY. 1996. Regulatory effects of cellular nicotinamide nucleotides and enzyme activities on poly(3-hydroxybutyrate) synthesis in recombinant Escherichia coli. Biotechnol. Bioeng. 52: 707-712.

28. Li ZJ, Cai L, Wu Q, Chen GQ. 2009. Overexpression of NAD kinase in recombinant Escherichia coli harboring the $p h b C A B$ operon improves poly(3-hydroxybutyrate) production. Appl. Microbiol. Biotechnol. 83: 939-947.

29. Shi F, Huan X, Wang X, Ning J. 2012. Overexpression of NAD kinases improves the L-isoleucine biosynthesis in Corynebacterium glutamicum ssp. lactofermentum. Enzyme Microb. Technol. 51: 73-80.

30. Wang X, Peng F, Dong G, Sun Y, Dai X, Yang Y, et al. 2018. Identification and validation of appropriate reference genes for qRT-PCR analysis in Corynebacterium glutamicum. FEMS Microbiol. Lett. 365(8): doi: 10.1093/femsle/fny030.

31. Wen S, Chen X, Xu F, Sun H. 2016. Validation of reference genes for real-time quantitative PCR (qPCR) analysis of Avibacterium paragallinarum. PLoS One 11: e0167736.

32. Sihto HM, Tasara T, Stephan R, Johler S. 2014. Validation of reference genes for normalization of qPCR mRNA expression levels in Staphylococcus aureus exposed to osmotic and lactic acid stress conditions encountered during food production and preservation. FEMS Microbiol. Lett. 356: 134-140. 
33. Xu N, Zheng Y, Wang X, Krulwich TA, Ma Y, Liu J. 2018. The Lysine 299 residue endows the multisubunit Mrp1 antiporter with dominant roles in $\mathrm{Na}^{+}$resistance and $\mathrm{pH}$ homeostasis in Corynebacterium glutamicum. Appl. Environ. Microbiol. 84: e00110-118.

34. Liu J, Fujisawa M, Hicks DB, Krulwich TA. 2009. Characterization of the functionally critical AXAXAXA and PXXEXXP motifs of the ATP synthase c-subunit from an Alkaliphilic Bacillus. J. Biol. Chem. 284: 8714-8725.

35. Lanzetta PA, Alvarez LJ, Reinach PS, Candia OA. 1979. An improved assay for nanomole amounts of inorganic phosphate. Anal. Biochem. 100: 95-97.

36. Kalinowski J, Bathe B, Bartels D, Bischoff N, Bott $\mathrm{M}$, Burkovski A, et al. 2003. The complete Corynebacterium glutamicum ATCC 13032 genome sequence and its impact on the production of L-aspartate-derived amino acids and vitamins. J. Biotechnol. 104: 5-25.

37. Reinscheid DJ, Schnicke S, Rittmann D, Zahnow U, Sahm H, Eikmanns BJ. 1999. Cloning, sequence analysis, expression and inactivation of the Corynebacterium glutamicum pta-ack operon encoding phosphotransacetylase and acetate kinase. Microbiology 145 (Pt 2): 503-513.

38. Yokota A, Terasawa Y, Takaoka N, Shimizu H, Tomita F. 1994. Pyruvic acid production by an F1-ATPase-defective mutant of Escherichia coli W1485lip2. Biosci. Biotechnol. Biochem. 58: 2164-2167.

39. Liu LM, Li Y, Du GC, Chen J. 2006. Increasing glycolytic flux in Torulopsis glabrata by redirecting ATP production from oxidative phosphorylation to substrate-level phosphorylation. J. Appl. Microbiol. 100: 1043-1053.

40. Aoki R, Wada M, Takesue N, Tanaka K, Yokota A. 2005. Enhanced glutamic acid production by a $\mathrm{H}^{+}$-ATPase-defective mutant of Corynebacterium glutamicum. Biosci. Biotechnol. Biochem. 69: 1466-1472.

41. Fillingame RH, Angevine CM, Dmitriev OY. 2003. Mechanics of coupling proton movements to c-ring rotation in ATP synthase. FEBS Lett. 555: 29-34.

42. Sekiya M, Nakamoto RK, Al-Shawi MK, Nakanishi-Matsui M, Futai M. 2009. Temperature dependence of single molecule rotation of the Escherichia coli ATP synthase F1 sector reveals the importance of gamma-beta subunit interactions in the catalytic dwell. J. Biol. Chem. 284: 22401-22410.

43. Becker J, Zelder O, Hafner S, Schroder H, Wittmann C. 2011. From zero to hero--design-based systems metabolic engineering of Corynebacterium glutamicum for L-lysine production. Metab. Eng. 13: 159-168.

44. Park SH, Kim HU, Kim TY, Park JS, Kim SS, Lee SY. 2014. Metabolic engineering of Corynebacterium glutamicum for L-arginine production. Nat. Commun. 5: 4618.

45. Jojima T, Fujii M, Mori E, Inui M, Yukawa H. 2010. Engineering of sugar metabolism of Corynebacterium glutamicum for production of amino acid L-alanine under oxygen deprivation. Appl. Microbiol. Biotechnol. 87: 159-165.

46. Bartek T, Blombach B, Zonnchen E, Makus P, Lang S, Eikmanns BJ, et al. 2010. Importance of NADPH supply for improved L-valine formation in Corynebacterium glutamicum. Biotechnol. Prog. 26: 361-371. 